\title{
Advance Directives as Support of Autonomy for Persons with Dementia? A Pilot Study among Persons with Dementia and Their Informal Caregivers
}

\author{
Martina Schmidhuber ${ }^{a}$ Sandra Haeupler ${ }^{b}$ Velislava Marinova-Schmidt ${ }^{b}$ \\ Andreas Frewer ${ }^{a}$ Peter L. Kolominsky-Rabas ${ }^{b}$ \\ anstitute for History of Medicine and Medical Ethics, Friedrich Alexander University

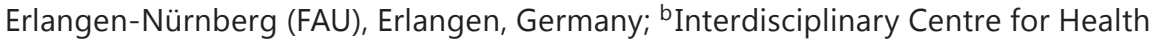 \\ Technology Assessment (HTA) and Public Health, Friedrich Alexander University \\ Erlangen-Nürnberg (FAU), Erlangen, Germany
}

\author{
Keywords \\ Dementia · Advance directives · Informal caregivers · Autonomy · Medical ethics
}

\begin{abstract}
Background: Advance directives could be an important instrument to support a person's will once he/she is not able to consent anymore - if composed competently. A survey was conducted to identify the level of knowledge concerning possibilities and limits of advance directives. Methods: The study was conducted as part of the Bavarian Dementia Survey (BayDem). Data were collected from January 2014 to December 2015 by structured face-to-face interviews. Study participants were persons with dementia and their informal caregivers $(n=74)$. Results: In total, $66 \%$ reported having written an advance directive. Concerning the participants' knowledge about possibilities and limitations of advance directives, a lack of knowledge was noted about the possibility to revoke an advance directive. Furthermore, $70 \%$ of informal caregivers and $56 \%$ of persons with dementia were not aware of the possibility to include dementia-specific terms in the advance directive. Conclusion: It is necessary to optimize structures for public information and education concerning the topic of advance directives for persons with dementia.




\section{Background}

Dementia is a general term used to refer to certain symptoms caused by specific diseases. The most common type of dementia is Alzheimer disease. At the moment, more than 46 million people worldwide are living with dementia. This number is estimated to increase to 131.5 million by 2050 [1]. Until now, there is no cure for dementia. Taking into account the physical, psychological and financial burdens [2], it is an enormous challenge for families and caregivers to deal with this disease. Considering these facts, it is necessary to support persons with dementia and their caregivers in coping with these challenges. In addition to pharmacological and nonpharmacological treatments, persons with dementia can be supported financially as well in their autonomous decision-making, which is one of the aims of this study. Persons with dementia should not feel disparaged as long as they are capable of making sensible decisions. Still, it needs to be borne in mind that there is a fine line between supporting the autonomy of persons with dementia and making them feel overburdened while being confronted with certain processes of decision-making. It seems very important to find the right measure and the adequate means to support persons with dementia in a suitable way [3].

Alzheimer's Disease International underlines the importance of supporting the autonomy of persons with dementia: they should be enabled to participate in the process of decisionmaking and have more options to control decisions regarding care and support, as well as decisions concerning what happens at the end of their lives [4]. It is important for persons with dementia to "consider possible future scenarios, and record their wishes and preferences at an early stage in the dementia process, while they still retain 'decision making capacity"' [4]. This kind of planning ahead is called "advance care planning" [5]. Advance care planning was first mentioned in the United States in the 1960s and since the 1990s, it has developed more and more worldwide [4]. The disease-specific ethical issue "dealing with the need for advance care planning" is mentioned in a number of national clinical practice guidelines for dementia [6].

As an instrument which aims to support the autonomy of persons with dementia, advance directives are an integral part of advance care planning. Prior to advanced dementia and well before a person has no longer the capacity to make end-of-life decisions, those affected should define how they want to be treated at the end of life. According to the Alzheimer's Association, advance directives are "legal papers that specify the type of medical care a person wants to receive once he or she no longer has capacity to make such decisions, and who should be in charge of making those decisions" [7]. The advance directive should be in written form and signed personally [8]. Ideally, the family doctor gives advice to the person who wants to write an advance directive. Due to time constraints and the lack of financial incentives, this assistance unfortunately does not occur very often. It is recommended to update the advance directive every 2 years and to also frame specific situations. An advance directive can only be helpful for doctors during the medical treatment of persons with dementia when specific terms are used. If the terms in the advance directive are not clear and precise enough (e.g., "I do not want life-extending measures"), the medical team might even be more challenged during the process of decision-making. Such a specific phrase to be used could be the following: "I do not want artificial nutrition in the last stage of dementia." Definitions like this are useful; however, it is still necessary to clarify if an advance directive is appropriate for a specific medical situation $[9,10]$. In this context, it seemed necessary to ask what persons with dementia and their informal caregivers actually know about the prospects and legal limits of advance directives. This research question was therefore explored in a pilot study in the European metropolitan region of Nuremberg (Germany). 
As the ethical discussion about advance directives and dementia was the background of this survey, the following questions outline the main focus of the discussion: How can advance directives support the autonomy of persons with dementia? And: Up to which stage of the disease should or could it be possible to revoke an advance directive? There obviously is a lack of empirical foundation concerning these research questions. In order to be able to address the question of autonomy of persons with dementia, these people have to be personally included in empirical research. Furthermore, the relationship between the ethical and practical implications of empirical findings might be very helpful to both outline the subject of strengthening autonomy for this specific group of people and point at open research questions yet to be dealt with.

\section{Methods}

The survey was conducted as part of the Bavarian Dementia Survey (BayDem), which is an ongoing study in Bavaria, Germany [11]. Data collection was performed from January 2014 to December 2015. Study participants were persons with dementia and their informal caregivers. The pilot study was approved by the local Ethics Committee of the Friedrich Alexander University Erlangen-Nuremberg, Germany. Eligibility criteria included: (1) person with dementia: dementia diagnosis according to ICD-10 (F00-F03), mild to moderate stage of dementia, capability to understand questions about advance directives and living in a homebased care setting within the metropolitan area of Nuremberg; (2) informal caregiver: primary responsibility as an informal caregiver for a person with diagnosed dementia, older than 18 years.

The study participants were selected from different forms of care facilities: from general practices, district hospitals, nursing homes, and counselling institutions. All study participants were informed about the objectives as well as about the scope of the study and provided written, informed consent for participation. Data were collected in written and structured face-to-face interviews based on a questionnaire. The questionnaire was designed by two scientists in Public Health (S.H., P.K.R.) and two scientists in Medical Ethics (M.S., A.F.). In addition, the questionnaire was discussed and refined within an interdisciplinary team of the Emerging Fields Initiative (EFI) Project "Human Rights in Healthcare" of the Friedrich Alexander University Erlangen-Nuremberg, which consisted of the following professionals: lawyers, ethicists, political scientists, and physicians.

The questionnaire consisted of two similar parts - one for the person with dementia and one for the informal caregiver. It included the following 5 main topics: (1) information acquisition about advance directives: the first section included structured questions about the choice of source of information concerning the topic "advance directives" (e.g., Internet, medical professionals, family,...); (2) reasons for/against an advance directive: in the second section, participants were asked for their main reasons to write an advance directive based on structured items; (3) difficulties in writing an advance directive: the comprehensibility of the process of writing an advance directive was assessed via a 5-point Likert scale from $1=$ very easy to 5 = very difficult; (4) knowledge about possibilities and limitations of advance directives: in order to find out more about the participants' knowledge concerning the possibilities and functions of an advance directive, participants had to rate 5 statements about advance directives (possible answers: yes; no; I don't know); (5) benefits and concerns regarding an advance directive: benefits and concerns regarding an advance directive were captured by open-ended questions.

Additionally, sociodemographic and medical parameters (diagnosis, Mini-Mental State Examination [12], comorbidities [Charlson Index] [13], caregiver burden [Burden Scale for 
Family Caregivers] [14]) were captured. The interviews were led by two trained experts, both with the professional background of Psychogerontology. The interviews lasted on average 20 $\mathrm{min}$. For some of the persons with a diagnosis of dementia, it was no longer possible to answer the questions. In those cases, "not reported" was entered and those persons were excluded from further analysis (see also the section Limitations). For data analysis, SPSS statistics 22.0 software was used.

\section{Results}

A total of 53 dyads (persons with dementia and principal informal caregivers) took part in the study. Thirty-two of the persons with dementia were excluded due to the severity of their stage of dementia or difficulties in understanding the survey questions. Ultimately, 53 interviews with informal caregivers (information about the advance directive of the person with dementia) and 21 interviews with people affected by dementia were conducted $(n=74)$. The interviewed persons with dementia were aged 45-92 years with a mean age of 74 years ( $S D=10) .78 \%$ live together with their informal caregiver, whereas $7 \%$ live alone and $15 \%$ live together with other persons. According to the results of the Mini-Mental State Examination, $79 \%$ of persons with dementia were in the mild stage and $21 \%$ in the moderate stage of dementia. Most of the persons were diagnosed with Alzheimer disease (50\%) or unspecified dementia (40\%). The informal caregivers were aged 31-89 years with a mean age of 64 years $(S D=14)$. The majority of informal caregivers were spouses $(63 \%)$ or children $(28 \%)$ of the person with dementia. Half of them (52\%) were retired; one third (32\%) were employed besides providing informal care. In total, 30\% of informal caregivers reported a moderate to severe caregiver burden. Participant characteristics of the persons with dementia and informal caregivers are summarized in Table 1.

A total of $85 \%$ of persons with dementia $(n=45)$ have at least thought about the possibility of an advance directive. According to answers from informal caregivers, two thirds $(n=35,66 \%)$ of the persons with dementia had written an advance directive. In total, $74 \%$ of them $(n=26)$ had written their advance directive prior to the dementia diagnosis.

Regarding age, gender, or education, there are no differences between study participants with or without an advance directive $(p>0.05)$. However, our results indicate a significant difference considering the informal caregivers' relation (spouse or children) to the person with dementia ( $p=0.008)$ : if the informal caregiver is a spouse, persons with dementia have a significantly higher chance of having written an advance directive.

\section{Information about Advance Directives}

For persons with dementia as well as for informal caregivers, the main sources of information for an advance directive are their families (informal caregivers: 16\%; persons with dementia: $26 \%$ ) and personal contact with medical professionals (informal caregivers: 16\%; persons with dementia: 26\%). Furthermore, the Internet (informal caregivers: $16 \%$, persons with dementia: $13 \%$ ) is used to search for information about advance directives. Within the group of informal caregivers, results indicated that children of the person with dementia are using the Internet significantly more often as a source of information than spouses $(p<0.05)$.

\section{Reasons for/against an Advance Directive}

Both the person with dementia and informal caregivers reported the following three main reasons for writing an advance directive: the possibility of self-determination $(22 \%)$, the wish to avoid undesired misery by therapies (20\%) as well as the preservation of self- 
Table 1. Characteristics of study participants

$\begin{array}{ll}\begin{array}{l}\text { Person with } \\ \text { dementia }(n=21)\end{array} & \text { Informal } \\ \text { caregiver }(n=53)\end{array}$

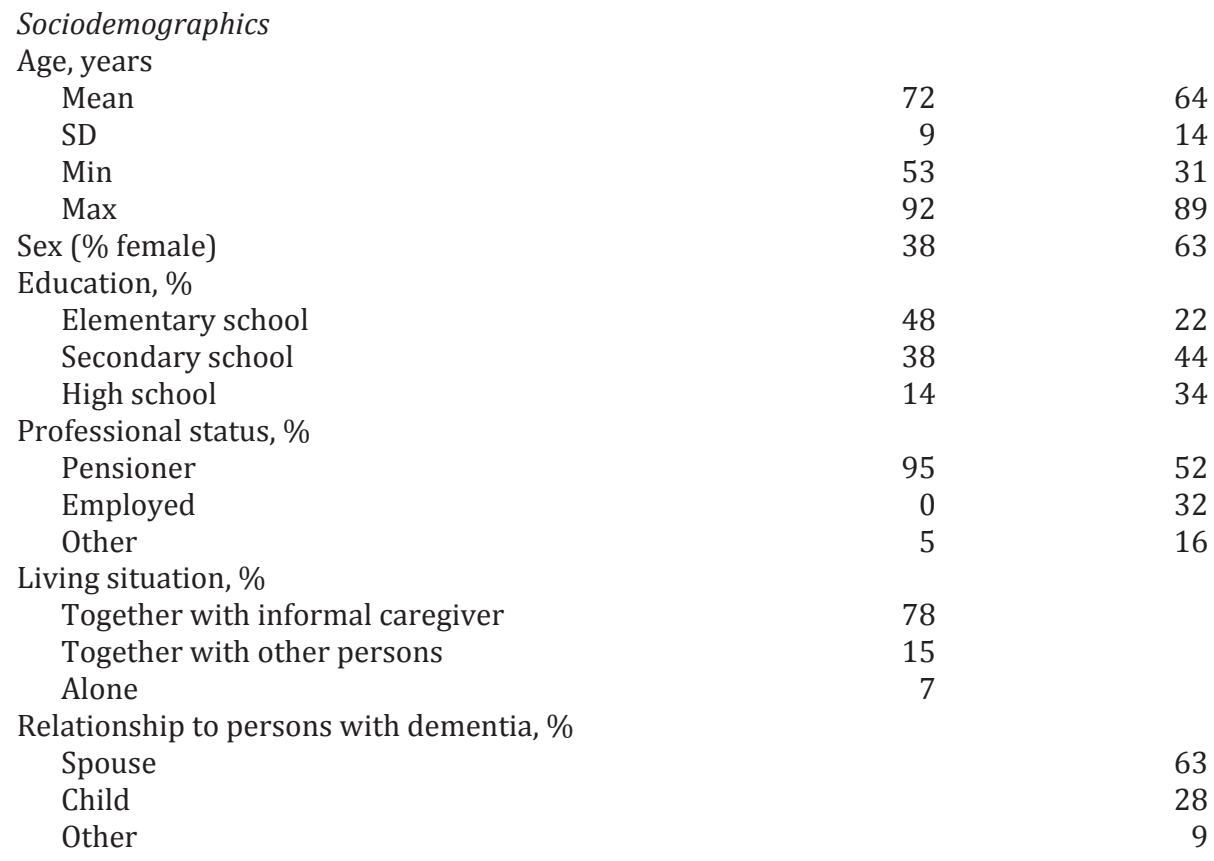

Medical parameters

MMSE stage, $\%$

Mild stage 79

Moderate stage 21

Severe stage 0

ICD diagnosis, $\%$

F00: Dementia in Alzheimer disease 50

F02: Dementia in other diseases classified elsewhere 10

F03: Unspecified dementia $\quad 40$

Comorbidities (Charlson Index), \%

None $\quad 56$

Mild to moderate $\quad 30$

Severe 14

Caregiver burden, \%

Mild $\quad 70$

Moderate $\quad 17$

Severe 13

determination during the end-of-life period (20\%). The main reason for not using the instrument of an advance directive $(n=18)$ was the fact that participants had never even thought about this instrument and therefore never considered it as an option.

\section{Difficulties in Writing an Advance Directive}

Participants rated the process of writing an advance directive (concerning comprehensibility) positively. In total, $59 \%$ rated the process as "very easy to understand" and $32 \%$ as "easy to understand" (mean $=1.50, \mathrm{SD}=0.7$ ). $8 \%$ indicated "partly easy, partly difficult." 
Table 2. Knowledge about possibilities and limitations of advance directives

\begin{tabular}{|c|c|c|c|}
\hline & Yes & No & Do not know \\
\hline \multicolumn{4}{|c|}{ Doctor is bound by advance directives, $\%$} \\
\hline Persons with dementia & $77^{\mathrm{a}}$ & 23 & 0 \\
\hline Informal caregivers & $94^{\mathrm{a}}$ & 2 & 4 \\
\hline \multicolumn{4}{|c|}{ Advance directives cannot be revoked, \% } \\
\hline Persons with dementia & 30 & $41^{\mathrm{a}}$ & 29 \\
\hline Informal caregivers & 22 & $71^{\mathrm{a}}$ & 7 \\
\hline \multicolumn{4}{|c|}{ Informal caregivers can revoke advance directives, \% } \\
\hline Persons with dementia & 35 & $30^{\mathrm{a}}$ & 35 \\
\hline Informal caregivers & 9 & $67^{\mathrm{a}}$ & 24 \\
\hline \multicolumn{4}{|c|}{ Advance directives image my volition, $\%$} \\
\hline Person with dementia & $88^{\mathrm{a}}$ & 0 & 12 \\
\hline Informal caregivers & $100^{\mathrm{a}}$ & 0 & 0 \\
\hline \multicolumn{4}{|c|}{ Possibilities of dementia-specific terms, $\%$} \\
\hline Person with dementia & $31^{\mathrm{a}}$ & 13 & 56 \\
\hline Informal caregivers & $17^{\mathrm{a}}$ & 13 & 70 \\
\hline
\end{tabular}

Informal caregivers $(n=46)$, person with dementia $(n=17)$. ${ }^{\text {a }}$ Correct answer.

\section{Knowledge about Possibilities and Limitations of Advance Directives}

In order to gather participants' knowledge about the possibilities and limitations of advance directives, participants had to rate 5 statements (Table 2). The first question was if a physician is bound to follow an advance directive by law: in total, $77 \%$ of persons with dementia and $94 \%$ of informal caregivers knew that a physician is legally bound by an advance directive. However, some indicated the wrong answer or were unsure about the answer. The next questions referred to the context of revocation. One of the questions was, for example, whether informal caregivers can revoke an advance directive. One third of the persons with dementia and two thirds of informal caregivers answered this correctly.

It is crucial to mention that $88 \%$ of the patients with dementia and $100 \%$ of the informal caregivers were aware of the fact that advance directives are intrinsically aimed at representing the will of the person who wrote it, especially in case of not being able to consent anymore. This is related to the results of the reasons for writing an advance directive: it definitely seems to be crucial to preserve as much self-determination as possible during every stage of this illness and until the end of life. In contrast, only $31 \%$ of persons with dementia and $17 \%$ of informal caregivers knew that there is the possibility to include dementia-specific terms within an advance directive. For this last question, both groups showed the highest uncertainty. Table 2 provides a detailed overview of participants' rating on the possibilities and limitations of advance directives.

\section{Benefits and Concerns Regarding an Advance Directive}

Participants indicated the following major benefits of advance directives: self-determination for end-of-life decisions $(n=20)$, decision support for end-of-life decisions for the informal caregiver $(n=11)$, to determine the person who will take care about my living will (informal caregiver) $(n=5)$, and to avoid undesired misery by therapies $(n=4)$. In contrast, one of the major concerns was that, despite of the availability of an advance directive, the will of the person with dementia will not be considered $(n=7)$. Another concern addresses the adequacy of the concrete medical context in combination with the expressed will. The participants feared that if the advance directive does not fully match 
the clinical and medical situation, it might therefore not be considered ( $n=3$ ). Additionally, 2 participants indicated that they were not able to decide for their future end-of-life situations at the moment.

\section{Discussion}

Our pilot study provides first detailed insights into the knowledge and use of advance directives in cases of diagnosed dementia. The results show, inter alia, that the participants of the test group are uncertain about the concrete possibilities and limitations of advance directives. This especially applies to the implementation of dementia-specific terms in advance directives. Only $31 \%$ of persons with dementia and $17 \%$ of informal caregivers knew that the possibility of including dementia-specific terms within an advance directive even exists. One reason may be that the majority of the people tend to write their advance directive prior to a dementia diagnosis - and are therefore not considering specific circumstances. In this context, our results highlight the need to provide more information specifically for the case of dementia, for example, avoiding tube feeding or avoiding antibiotics. Due to the fact that $74 \%$ of the participants wrote their advance directive prior to the dementia diagnosis, the particular time of the first dementia diagnosis seems to be a very important instant where the physician should also inform about dementia-specific terms for advance directives. Since advance directives can only be legally effective when they are written while the person with dementia still has legal capacity, it is highly important as well as recommended to complete advance directives as soon as possible following the diagnosis of dementia [7]. The survey shows that neither age nor gender nor education has a significant influence on the fact of having prepared and written an advance directive. Yet, there is a significant correlation between informal caregivers' relation to the person with dementia and having an advance directive: if the informal caregiver is a spouse, more persons with dementia have an advance directive. Other studies show similar results concerning the caregivers' relation to the person with dementia. There is, for example, a study about terminally ill patients and advance directives that shows that patients are significantly more likely to have an advance directive if the caregiver is older [15]. It could be considered a logical conclusion that an affected spouse who is of advanced age is more likely to consider an advance directive for him or herself - and will therefore be more aware of this subject and better informed than, for example, the children of the person with dementia. The issue of an advance directive is obviously more relevant for elderly people than for younger ones like children or other descendants.

\section{Revocation and Autonomy}

While the participants were quite aware of the fact that an advance directive is legally binding ( $77 \%$ of persons with dementia, $94 \%$ of informal caregivers), there still were some insecurities about the possibility of revocation (only $41 \%$ of persons with dementia and $71 \%$ of informal caregivers knew that an advance directive can be revoked). In this context, also ethical questions arise: How long should a revocation of a person with dementia be accepted? When is he or she not autonomous anymore? Who should decide in place of the affected person in this difficult situation? Which expressions - verbal, mimic or gesticulatory - should be taken into account as expressions of an autonomous will? There are no general answers to those questions. Sometimes, a person at a later stage of dementia has some mentally clear moments. If in such moments this person states the wish to revoke his or her advance directive several times, it should be taken seriously. One could also ask if only a verbal revocation should be considered, or also gestures and mimic expressions $[3,16]$. In many cases of late stages of dementia, only gestures and mimic expressions are possible. One position is that 
these expressions are as important as the verbal ones [17]. Others claim that these kinds of expressions are not expressions of autonomy anymore, but rather merely "vegetative life signs" [18]. It is very important to decide carefully from case to case and to consider the specific context with the whole medical team: doctors, caregivers, and relatives should decide together how to proceed. If the situation is absolutely unclear, it is regarded as imperative to establish an "in dubio pro vita" attitude.

\section{Personal Identity and Autonomy}

There is no general point in dementia where it can be stated that autonomy is lost. Autonomy is not an all-or-nothing capacity. It decreases gradually during the disease [19]. Concerning this topic, there is a broad discussion in medical ethics addressing questions about personal identity [20-23]. Is the person with dementia the same as he/she used to be before? And is the person with dementia entitled to revoke the advance directive that he/she had written before the diagnosis of dementia $[21,22]$ ? There are basically three main positions related to the question of personal identity: with recourse to the philosopher John Locke, some say that memory is one of the most important aspects of personal identity. On the other hand, some emphasize the bodily existence of human beings and state that persons are more than their memory [24]. Others prefer the narrative view, which refers to the whole life as a story. According to this view, dementia would also be part of this story and hence part of personal identity [25]. This position seems to be the most promising one to deal with the individual questions concerning persons with dementia - especially those questions addressing the subject of autonomy. Sometimes in a life story, especially in vulnerable situations like dementia, people do not want to be sole authors of their own life stories or are not able to actively tell them anymore - and need support at the end of life [26]. Concerning advance directives, for example, the authors might need other persons to interpret them at some point. Even if a person has written an advance directive autonomously, this document has to be read, interpreted and implemented by other persons in case of loss of capacity to consent. This highlights the fact that autonomy is relational, especially in situations of vulnerability. Supporting autonomy of persons with dementia can therefore also mean providing the best measures for interpretation (for the informal caregivers) as well as for expressing oneself (for the person with dementia) by improving the standards of information and implementing dementia-specific terms in advance directives.

\section{Level of Information and How to Increase It}

The results indicate that there is a general lack of specific knowledge about advance directives. As reported above, the most rated reason for not having an advance directive is the fact that people never even thought about this instrument. On the other hand, our results indicate that once a person decides to have an advance directive, the process of writing an advance directive itself is not a hurdle: almost two thirds of the study group in total perceived the process as "easy to understand." Therefore, one of the main problems concerning advance directives seems to be the perceptibility of this instrument, not the instrument itself. One aspect that could address this issue is to increase public awareness. It is necessary to inform people at an advanced age more adequately and specifically concerning this issue. For persons with dementia, it is necessary to know that dementia-specific terms should be added in their advance directive, for example, end-of-life decisions like tube feeding or antibiotic treatment. For younger informal caregivers, it is important to have adequate Internet sources for information about advance directives in dementia. Medical professionals are one of the most important information sources in medical matters for senior citizens (informal caregivers: $16 \%$; persons with dementia: $26 \%$ ). Consequently, it seems to be promising, as already stated above, to involve those medical professionals who made the first diagnosis of dementia in 
informing their patients about possibilities and limitations of advance directives. Therefore, more education and training in ethics and law for doctors is needed for this kind of support [27].

In addition, it could be helpful to inform people about the legal parameters of the advance directive with booklets in easy language and to give the possibility to ask if there are ambiguities.

\section{Limitations}

Although our pilot study provided essential insights into the perspectives of persons with dementia and their informal caregivers, some limitations need to be taken into account. First, structured questions can have a suggestive impact; some answers may be given because they are enunciated, even though the persons actually did not think about this issue before. In addition, the sample size and structure have to be taken in account when interpreting the results. Due to the stage of the disease, we had to exclude 32 persons with dementia; however, we still received answers from their informal caregivers. In this context, it could be promising to conduct further surveys about the individual capacities concerning the ability to answer such questions - and if it would therefore be necessary or possible to adjust the mode of questioning, or if those limitations could allow conclusions about the individual point where handling an advance directive autonomously becomes too difficult.

\section{Conclusion}

The results of this survey clearly illustrate that, especially for persons with dementia, the wishes to preserve self-determination and to have one's own autonomous will, respected even in case of not being able to consent anymore, are the most important reasons for writing an advance directive. Concerning the subject of advance directives, the results show a certain level of rather unspecific general knowledge, but also a large amount of insecurities. Those insecurities display the unpredictability and the difficulties in dealing with dementia, which have been shown in the Discussion - for the affected persons as well as for informal caregivers and medical teams involved in treatment. The aim of this study and the ethical considerations in this article were to point out that advance directives can be a powerful instrument to deal with those insecurities and to strengthen the autonomy of persons with dementia - as long as they are being used competently by all the parties involved. For that, more specific information is needed to support the perceptibility of this instrument. It would also be advisable to develop advance directives especially for persons with dementia with dementia-specific terms. If preprinted forms in easy language existed, including anticipated dementia-specific situations, it would clearly reduce the obstacles of writing an advance directive, and if the family doctor helped people and their caregivers to fill out the form and explain the specific situations that might occur, then most of the insecurities could be prevented for everybody concerned. Finally, more research about the knowledge as well as about the insecurities of people is needed to be able to develop adequate strategies to be used in practice.

\section{Acknowledgements}

We express our special thanks to all persons with dementia and informal caregivers who participated in our study. Furthermore, we would like to thank the Emerging Fields Initiative Project "Human Rights in Healthcare (EFI)" Consortium. 
We also thank our native speaker R.D.H. for proof reading and our colleague C.H. for reviewing the article critically.

We acknowledge support by the Deutsche Forschungsgemeinschaft and Friedrich-Alexander-Universität Erlangen-Nürnberg (FAU) within the funding pogramme Open Access Publishing.

\section{Statement of Ethics}

The study was approved by the Ethics Committee of the Friedrich Alexander University Erlangen-Nuremberg (Germany).

\section{Disclosure Statement}

The authors declare that they have no conflicts of interest to disclose.

\section{Funding Sources}

This study was initiated by the Bavarian State Parliament and data collection was funded by the Bavarian Ministry of Health and (StMGP) (grant No. G42b-G8092.9-2014/10-7). The research work was funded within the EFI Project "Human Rights in Healthcare" as part of the EFI Project "Human Rights in Healthcare" of the Friedrich Alexander University ErlangenNürnberg (FAU).

\section{Author Contributions}

All authors designed the study and the questionnaire. S.H. analyzed and interpreted the data; M.S. analyzed the implied ethical aspects. S.H. and M.S. contributed equally to the study. V.M.-S. conducted interviews with persons with dementia and informal caregivers. A.F. and P.L.K.-R. supervised the study and provided input for the interpretation and discussion of results. All authors read and approved the final manuscript.

\section{References}

1 Alzheimer's Disease International: World Alzheimer Report 2015: The Global Impact of Dementia. An Analysis of Prevalence, Incidence, Cost and Trends. London, Alzheimer's Disease International, 2015.

$\rightarrow 2$ Alzheimer's Association: 2016 Alzheimer's disease facts and figures. Alzheimers Dement 2016;12:459-509.

3 Frewer A, Bergemann L, Schmidhuber M (eds): Demenz und Ethik in der Medizin. Standards zur guten klinischen Praxis. Jahrbuch Ethik in der Klinik (JEK). Würzburg, Königshausen \& Neumann, 2015, vol 8.

4 Alzheimer's Disease International: World Alzheimer Report 2013: Journey of Caring. An Analysis of LongTerm Care for Dementia. London, Alzheimer's Disease International, 2013.

5 Coors M, Jox RJ, in der Schmitten J (eds): Advance Care Planning. Von der Patientenverfügung zur gesundheitlichen Vorausplanung. Stuttgart, Kohlhammer, 2015.

-6 Knüppel H, Mertz M, Schmidhuber M, Neitzke G, Strech D: Inclusion of ethical issues in dementia guidelines: a thematic text analysis. PLoS Med 2013;10:1-11.

7 https://www.alz.org/national/documents/brochure_endoflifedecisions.pdf (accessed October 24, 2016).

8 Wiesing U, Jox RJ, Hessler HJ, Borasio GD: A new law on advance directives in Germany. J Med Ethics 2010;36: 779-783.

9 Frewer A, Fahr U, Rascher W (eds): Patientenverfügung und Ethik. Beiträge zur guten klinischen Praxis. Jahrbuch Ethik in der Klinik (JEK). Würzburg, Könighausen \& Neumann, 2009, vol 2. 
10 May AT, Kress H, Verrel T, Wagner T (eds): Patientenverfügungen. Handbuch für Berater, Ärzte und Betreuer. Berlin, Springer, 2016.

11 Schaller S, et al: Bayerischer Demenz Survey: Methodik einer Längsschnitterhebung zur Versorgungssituation bei Demenz. Gesundheitswesen 2015;77:A370.

12 Folstein MF, Folstein SE, McHugh PR: "Mini-mental state". A practical method for grading the cognitive state of patients for the clinician. J Psychiatr Res 1975;12:189-198.

13 Charlson ME, Pompei P, Ales KL, MacKenzie CR: A new method of classifying prognostic comorbidity in longitudinal studies: development and validation. J Chronic Dis 1987;40:373-383.

14 Graessel E, Berth H, Lichte T, Grau H: Subjective caregiver burden: validity of the 10-item short version of the Burden Scale for Family Caregivers BSFC-s. BMC Geriatr 2014;14-23.

15 Ho G, et al: Patient and caregiver characteristics related to completion of advance directives in terminally ill patients. Palliat Support Care 2017;15:12-19.

16 Foster C, Herring J, Doron I (eds): The Law and Ethics of Dementia. Oxford, Hart Publishing, 2014.

17 Dabrock P: Formen der Selbstbestimmung. Theologisch-ethische Perspektiven zu Patientenverfügungen bei Demenzerkrankungen. Z Med Ethik 2007;53:127-144.

18 Deutscher Ethikrat: Demenz und Selbstbestimmung. Stellungnahme. Berlin, 2012.

19 Schmidhuber M: Der Stellenwert von Autonomie für ein gutes Leben Demenzbetroffener. Salzburger Beiträge zur Sozialethik 5. Salzburg, 2013.

20 Birnbacher D: Patientenverfügungen und Advance Care Planning bei Demenz und anderen kognitiven Beeinträchtigungen. Ethik Med 2016;4:283-294.

21 Dresser R: Dworkin on dementia. Elegant theory, questionable policy; in Green SA, Bloch S (eds): An Anthology of Psychiatric Ethics. New York, Oxford University Press, 2006, pp 297-301.

22 Dworkin R: Autonomy and the demented self; in Green SA, Bloch S (eds): An Anthology of Psychiatric Ethics. New York, Oxford University Press, 2006, pp 293-296.

23 Demarco JP, Lipuma SH: Dementia, advance directives, and discontinuity of personality. Camb Q Healthc Ethics 2016;25:674-685.

-24 Coors MA: Dementalized body? Reconsidering the human condition in the light of dementia. Geriatr Ment Health Care 2013;1:34-38.

25 Schechtman M: The Constitution of Selves. Ithaca, Cornell University Press, 2007.

26 Davis JK: Precedent autonomy, advance directives, and end-of-life care; in Steinbock B (ed): The Oxford Handbook of Bioethics. Oxford, Oxford University Press, 2007, pp 349-374.

27 White BP, Willmott L, Williams G, Cartwright C, Parker M: The role of law in decisions to withhold and withdraw life-sustaining treatment from adults who lack capacity: a cross-sectional study. J Med Ethics 2017;43:327333. 